emails on their office systems, and submit to a full background check and polygraph every five years. More importantly, they are fully aware that they are targets of foreign intelligence services that will be watching for any weakness that can be exploited. When I was at NSA, we all knew that a government was likely listening to all our phone calls-just not the U.S. government.

Every time an espionage incident happensJohn Walker, Aldrich Ames, Bradley Manning, Edward Snowden-in which someone with a clearance has signed an oath to not disclose information and then violates that oath and trust, it comes as a disappointment, a feeling of betrayal by someone in the family. It hurts NSA and it hurts the country. We are all aware that untrustworthy individuals will continue to crop up, but it's still a shock. System administrators occupy key positions today, much as crypto custodians, the holders of the keys (Walker), did years ago. The damage that such a person can cause to an agency is immense.
The trust that is damaged, both within our own country and with other countries as well, will take years to rebuild.

Finding intelligence in the mountain of data that exists today is a monumental task. If it was simple, we would predict all the terrible events that happen-9/11, Boston Marathon bombing, etc.-but it's not. There is much publicity on events that happen and none on those that are stopped. NSA is just one player on the team-from the Department of Homeland Security, FBI, Drug Enforcement Administration, Coast Guard, Air Force, Army, Navy, Marines, to local police and fire departments-whose reason for existence is to protect the people of this nation. Events like the Snowden leaks make that effort harder.

Whether the rules should be changed further is a great topic for debate. What is already clear, however, is that the threats are not easing. And they are not going to go away.

\title{
Lusztig Awarded Shaw Prize
}

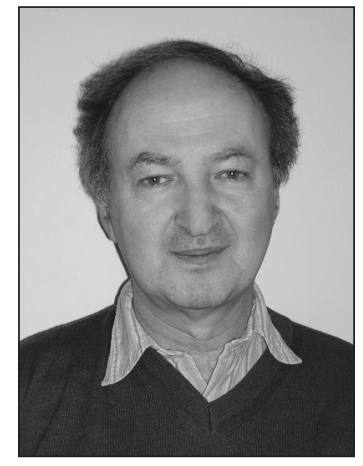

George Lusztig
On May 27, 2014, the Shaw Foundation announced the awarding of the 2014 Shaw Prize in Mathematical Sciences to GeORge Lusztig of the Massachusetts Institute of Technology "for his fundamental contributions to algebra, algebraic geometry, and representation theory and for weaving these subjects together to solve old problems and reveal beautiful new connections." The prize carries a cash award of US\$1 million.

The Shaw Prize in Mathematical Sciences Committee released the following statement about Lusztig's work.

"For more than two hundred years, symmetry groups have been at the center of mathematics and its applications: in Fourier's work on the heat equation in the early 1800s; in Weyl's work on quantum mechanics in the early 1900s; and in the approach to number theory created by Artin and Chevalley. These classical works show that answers to almost any question involving a symmetry group lie in understanding its realizations as a group of matrices; that is, in terms of its representations.

DOI: http://dx.doi.org/10.1090/noti1154
"Starting with his early work in the 1970s and 1980s, in part jointly with Deligne, Lusztig gave a complete description of the representations of finite Chevalley groups, these being the building blocks of finite symmetry groups. The Deligne-Lusztig description uses the topology and geometry of Schubert varieties. The latter were introduced in the nineteenth century as a tool to count solutions of algebraic equations.

"The vision of this work is that the algebraic subtleties of representation theory correspond perfectly to the geometric/topological subtleties of Schubert varieties. This vision has grown into a broad and powerful theme in Lusztig's work: he has shown that many central problems in representation theory-including those of real and $p$-adic Lie groups, which are the language of applications from number theory to mathematical physics - can be related to topology and geometry by means of Schubert varieties. This idea is at the heart of many exciting recent developments, for example in progress toward the Langlands programme in automorphic forms.

"Representations are complicated, as are the Schubert varieties to which they are related. Beginning in a 1979 paper with David Kazhdan, and 
continuing through his most recent work, Lusztig has found combinatorial tools to describe their topology and geometry. (These tools are easy to describe, but had not been used previously in mathematics.) His ideas have guided and inspired the development of perverse sheaves, a tool for studying the topology of general singular algebraic varieties.

"These tools, in the hands of Lusztig and of hundreds of other mathematicians, have made possible a depth of understanding of representations and of Schubert varieties that was unimaginable before his work."

George Lusztig was born in 1946 in Timisoara, Romania. He received his Ph.D. in 1971 from Princeton University under the direction of William Browder. He taught at the University of Warwick from 1974 to 1977 , then joined the faculty of the Massachusetts Institute of Technology, where he was Norbert Wiener Professor from 1999 to 2009 and is currently Abdun-Nur Professor of Mathematics. He received the Cole Prize (1985) and the Steele Prize for Lifetime Achievement (2008) from the AMS. He has also been a recipient of the Berwick Prize of the London Mathematical Society (1977) and the Brouwer Medal of the Dutch Mathematical Society (1999). He is a fellow of the Royal Society (1983) and of the American Academy of Arts and Sciences (1991) and a member of the National Academy of Sciences (1992).

The Shaw Prize is an international award es tablished to honor individuals who are currently active in their respective fields and who have achieved distinguished and significant advances, who have made outstanding contributions in culture and the arts, or who have achieved excellence in other domains. The award is dedicated to furthering societal progress, enhancing quality of life, and enriching humanity's spiritual civilization. Preference is given to individuals whose significant work was recently achieved.

The Shaw Prize consists of three annual awards: the Prize in Astronomy, the Prize in Science and Medicine, and the Prize in Mathematical Sciences. Established under the auspices of Run Run Shaw in November 2002, the prize is managed and administered by the Shaw Prize Foundation based in Hong Kong.

Previous recipients of the Shaw Prize in Mathematical Sciences are David L. Donoho (2013), Maxim Kontsevich (2012), Demetrios Christodoulou and Richard S. Hamilton (2011), Jean Bourgain (2010), Simon K. Donaldson and Clifford H. Taubes (2009), Vladimir Arnold and Ludwig Faddeev (2008), Robert Langlands and Richard Taylor (2007), David Mumford and Wen-Tsun Wu (2006), Andrew Wiles (2005), and Shiing-Shen Chern (2004).

\section{- From Shaw Foundation announcements}

THE HONG KONG UNIVERSITY OF SCIENCE AND TECHNOLOGY

Faculty Position(s)

\section{Department of Mathematics}

The Department of Mathematics invites applications for tenure-track faculty positions at the rank of Assistant Professor in all areas of mathematics. Applications at the rank of Associate Professor or above may also be considered. Other things being equal, preference will be given to areas consistent with the Department's strategic planning.

Applicants should have a $\mathrm{PhD}$ degree and strong experience in research and teaching. Applicants with exceptionally strong qualifications and substantial experience in research and teaching may be considered for positions above the Assistant Professor rank.

Starting rank and salary will depend on qualifications and experience. Fringe benefits include medical/dental benefits and annual leave. Housing will also be provided where applicable. Initial appointment will be on a three-year contract, renewal subject to mutual agreement. A gratuity will be payable upon successful completion of the contract.

\section{Application Procedure}

Applications received on or before 31 December 2014 will be given full consideration for appointment in 2015. Applications received afterwards will be considered subject to the availability of positions. Applicants for Assistant Professor position should send their curriculum vitae together with at least three research references and one teaching reference to the Human Resources Office, HKUST, Clear Water Bay, Kowloon, Hong Kong. Applicants for Associate Professor position and above should send a curriculum vitae and the names of at least three research referees to the Human Resources Office.

More information about the University is available on the University's homepage at http://www.ust.hk.

(Information provided by applicants will be used for recruitment and other employment-related purposes.)
AMERICAN MATHEMATICAL SOCIETY MathSciNet

\section{Mathematical Reviews}

The premier gateway to scholarly literature in the mathematical sciences

Since 1940, Mathematical Reviews (MR) has served researchers and scholars in the mathematical sciences by providing timely information on peer-reviewed articles and books. The database of more than 3 million items contains reviews, abstracts, and bibliographic information for much of the world's mathematical sciences literature.

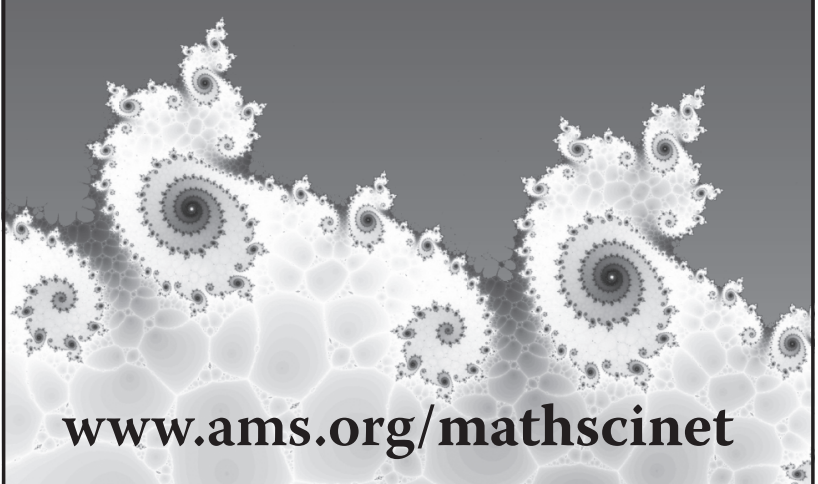

